\title{
Polarons in a Dipolar Condensate
}

\author{
Ben Kain ${ }^{1,2}$ and Hong Y. Ling ${ }^{2,3}$ \\ ${ }^{1}$ Department of Physics, College of the Holy Cross, Worcester, Massachussets 01610, USA \\ ${ }^{2}$ Department of Physics and Astronomy, Rowan University, Glassboro, New Jersey 08028, USA \\ ${ }^{3}$ Kavli Institute for Theoretical Physics, University of California, Santa Barbara, California 93106, USA
}

We consider a polaronic model in which impurity fermions interact with background bosons in a dipolar condensate. The polaron in this model emerges as an impurity dressed with a cloud of phonons of the dipolar condensate, which, due to the competition between the attractive and repulsive part of the dipole-dipole interaction, obey an anisotropic dispersion spectrum. We study how this anisotropy affects the Čerenkov-like emission of Bogoliubov phonon modes, which can be directly verified by experiments in which a dipolar BEC moves against an obstacle. We also study the spectral function of impurity fermions, which is directly accessible to the momentum resolved rf spectroscopy in cold atoms.

PACS numbers: 67.85.-d, 67.85.Pq, 71.38.Fp

\section{INTRODUCTION}

A conduction electron in an ionic crystal or a polar semiconductor displaces nearby ions, thereby polarizing the medium in the vicinity of the electron. An analogous picture emerges when an impurity atom is immersed in an ultracold atomic quantum gas containing atoms distinct from but capable of interacting with the impurity atom. The impurity finds itself surrounded by and traveling with its local disturbance, a cloud of background atoms, forming a polaron. In recent years, much effort has been focused on systems where both impurity and background atoms are fermions (see $[1,2]$ for a review), inspired by the remarkable agreement between theoretical predictions $[3,4]$ and experimental findings obtained from set-ups where a single spin- $\downarrow$ impurity is immersed in a sea of spin- $\uparrow$ background atoms [5, 6], a setting that shares much resemblance to the Kondo problem in condensed matter physics $[7,8]$. The present work, however, concerns polaron models where the impurity atoms are fermions but the background atoms are bosons in a Bose-Einstein condensate (BEC), in which density fluctuations of the BEC are described by phonons. Recent years have also witnessed an increased interest in such systems due largely to their similarity to the electronphonon system where the polaron picture $[9]$ is central to the understanding of colossal magnetoresistance materials [10] and is believed to play a vital role in the physics of high- $T_{c}$ superconductivity in strongly correlated materials [11-13] and in unconventional pairing mechanisms [14].

In this paper, motivated by recent experimental advancement in achieving dipolar quantum gases consisting of either heteronuclear molecules with electric dipoles $[15,16]$ or atoms with magnetic dipoles [17-20], we consider the aforementioned Bose polaron models except that the background atoms are now bosons in a dipolar condensate. In a dipolar quantum gas [21, 22], the dipole-dipole interaction represents a control knob inaccessible to nondipolar bosons. Thus, mixing dipolar bosons with fermions opens up new possibilities. An important consequence of the dipole-dipole interaction is that the phonon spectrum of a dipolar condensate is no longer isotropic - one can tune the dipolar interaction to lower the energy of a phonon along some directions while simultaneously increasing it along other directions [23], a phenomenon that a recent experiment demonstrated using Raman-Bragg spectroscopy in a dipolar chromium BEC [24]. Thus, impurity fermions submerged in a dipolar condensate act as anisotropic polarons, interacting with surrounding phonons. While many studies exist in the literature concerning the polaronic Bose-Fermi models, including those in Refs. [25-27] for large (continuous) polarons, those in Refs. $[28,29]$ for small (Holstein) polarons, and some experimental developments [30-32], to the best of our knowledge, none of them have seriously considered polaron systems with background atoms that are dipolar bosons. The purpose of our work here is to extend studies from nondipolar to dipolar Bose-Fermi polaron models and develop theoretical tools which allow us to gain, from investigations in the weak coupling limit, quantitative understanding of the impurity polarons in the dipolar condensate.

In Sec. II, we present the Hamiltonian for the effective polaronic model where density fluctuations of the dipolar condensate are described by phonons, and we derive, in the same section, the impurity self-energy, taking into consideration only single-phonon-impurity scattering processes. In Sec. III, we evaluate this self-energy on the mass shell and use it to gain some quantitative insight into the physics of the anisotropic polarons under consideration. A moving polaron may emit phonons in much the same way that a moving charge emits electromagnetic radiation or Čerenkov radiation when its velocity exceeds a certain threshold $[33,34]$. In addition to the effective mass, the self-energy will be used in Sec. III to analyze the decay rate of the polaron and to understand Čerenkov-like phonon emissions from an impurity in a dipolar condensate [35]. In Sec. IV, we describe polarons using Fermi liquid theory, focusing, however, on spectral functions, which can, in principle, be probed using 
the momentum resolved rf spectroscopy in cold atoms $[36,37]$. Finally, we conclude in Sec. V.

\section{THE EFFECTIVE HAMILTONIAN AND IMPURITY SELF-ENERGY}

Let us now turn to the specific model of a cold-atom mixture in which spin-polarized impurity fermions with mass $m_{F}$ permeate in a dipolar condensate of bosons with mass $m_{B}$. The condensate is confined in a sufficiently large trap so that it is practically homogeneous. In addition, all dipoles are assumed to point in the same direction as an external (either electric or magnetic) field, which we take to be the $z$ direction. The interactions in this model are divided into a short-range part and a long-range part. The former is described by $U_{B F}=4 \pi \hbar^{2} a_{B F} / m_{B F}\left[m_{B F}=2 m_{B} m_{F} /\left(m_{B}+m_{F}\right)\right]$ and $U_{B B}=4 \pi \hbar^{2} a_{B B} / m_{B}$, where $a_{B F}$ and $a_{B B}$ are, respectively, impurity-boson and boson-boson s-wave scattering lengths. The latter is described by $U_{D D}(\mathbf{q})=$ $8 \pi d^{2} P_{2}\left(z_{\mathbf{q}}\right)$, which is the dipole-dipole interaction between two bosons in momentum space, with $d$ the induced dipole moment, $P_{2}\left(z_{\mathbf{q}}\right) \equiv\left(3 z_{\mathbf{q}}^{2}-1\right) / 2$ the secondorder Legendre polynomial, where $z_{\mathbf{q}}=\cos \theta_{\mathbf{q}}$ and $\theta_{\mathbf{q}}$ is the angle between momentum $\hbar \mathbf{q}$ and the dipole direction (along the $z$ axis). To proceed, we limit our study to near zero temperature $(T \approx 0)$ where one can approximate, within the Bogoliubov approximation, the dipolar Bose gas as a uniform dipolar condensate of number density $n_{B}$ plus a collection of phonons (due to density fluctuations) that obey the dispersion spectrum [38, 39]

$$
\hbar \omega_{\mathbf{q}}=\hbar v_{B} q \sqrt{1+\left(\xi_{B} q\right)^{2}+2 \varepsilon_{d d} P_{2}\left(z_{\mathbf{q}}\right)},
$$

where $v_{B}=\sqrt{n_{B} U_{B B} / m_{B}}$ is the phonon speed in the absence of the dipolar interaction, $\xi_{B}=\hbar / \sqrt{4 m_{B} n_{B} U_{B B}}$ is the healing length, and $\varepsilon_{d d}=4 \pi d^{2} /\left(3 U_{B B}\right)$ measures the dipolar interaction relative to the contact interaction $U_{B B}$. As such, we are led to an effective Hamiltonian

$$
\begin{aligned}
\hat{H} & =\sum_{\mathbf{p}}\left(\xi_{\mathbf{p}}+n_{B} U_{B F}\right) \hat{a}_{\mathbf{p}}^{\dagger} \hat{a}_{\mathbf{p}} \\
& +\hbar \sum_{\mathbf{q} \neq 0} \omega_{\mathbf{q}} \hat{\beta}_{\mathbf{q}}^{\dagger} \hat{\beta}_{\mathbf{q}}+\frac{1}{\sqrt{V}} \sum_{\mathbf{q} \neq 0} g_{\mathbf{q}} \hat{\rho}_{\mathbf{q}}\left(\hat{\beta}_{\mathbf{q}}+\hat{\beta}_{-\mathbf{q}}^{\dagger}\right),
\end{aligned}
$$

which describes impurity fermions (of field operator $\hat{a}_{\mathbf{p}}$ and density operator $\left.\hat{\rho}_{\mathbf{q}}=\sum_{\mathbf{k}} \hat{a}_{\mathbf{q}+\mathbf{k}}^{\dagger} \hat{a}_{\mathbf{k}}\right)$ interacting with phonons (of field operator $\hat{\beta}_{\mathbf{q}}$ ) in a volume $V$. In Eq. (2), $\xi_{\mathbf{p}}=\epsilon_{\mathbf{p}}-\mu$ is the kinetic energy of a fermion, $\epsilon_{\mathbf{p}}=\hbar^{2} p^{2} / 2 m_{F}$, relative to the Fermi chemical potential $\mu$, and $g_{\mathbf{q}}=U_{B F} \sqrt{n_{B} \hbar q^{2} /\left(2 m_{B} \omega_{\mathbf{q}}\right)}$ is a momentumdependent coefficient measuring the impurity-phonon coupling strength. In solid state systems, phonon dispersion due to lattice vibration can be divided into an optical branch whose frequency remains almost independent of the wavenumber and an acoustic branch whose frequency

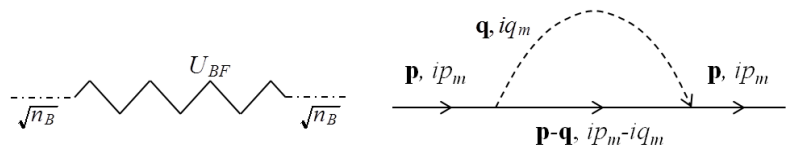

FIG. 1. Feynman diagrams for the self energy of an impurity (fermion) interacing with background bosons.

is linearly proportional to the wavenumber [40]. Equation (2) is the cold-atom analog of the Fröhlich Hamiltonian for electron-acoustic phonon systems since the Bogoliubov dispersion in Eq. (1) asymptotes to that of an acoustic phonon [41] in the limit of long wavelength. In the absence of the dipole-dipole interaction, Eq. (2) has been employed, within the context of cold atomic physics, to study polarons in the weak coupling limit [27, 42], under self-localization [25, 43, 44], and in the strongcoupling limit (using the Feynman path integral) [26, 45]. Having described our model in some detail, we now adopt a unit convention in which $\hbar=1$ is implied (unless keeping $\hbar$ helps to elucidate the physics).

The polaron effect arises from the impurity-polaron interaction which modifies the self-energy and hence the bare propagator of the impurity. Thus, much of the information about the properties of an impurity can be learned from its self-energy. At zero temperature, this self-energy can be conveniently obtained from the nonzero-temperature Matsubara results by setting $T=0$. In the weak coupling regime, the Matsubara self-energy for impurity fermions, up to second order in the BoseFermi interaction $U_{B F}$, reads

$$
\begin{aligned}
\Sigma\left(\mathbf{p}, i \omega_{n}\right) & =U_{B F} n_{B}-\frac{1}{\beta V} \sum_{\mathbf{q}, i q_{m}} g_{\mathbf{q}}^{2} \times \\
& D_{0}\left(\mathbf{q}, i q_{m}\right) G_{0}\left(\mathbf{p}-\mathbf{q}, i \omega_{n}-i q_{m}\right),
\end{aligned}
$$

where the first term is due to the interaction of the impurity fermions with the condensed bosons [Fig.1(left)], and the second term accounts for all single-phononimpurity scattering processes and can thus be read off directly from the Feynman diagram involving one phonon [Fig. 1(right)]. In Eq. (3), $G_{0}\left(\mathbf{p}, i \omega_{n}\right)=\left(i \omega_{n}-\xi_{\mathbf{p}}\right)^{-1}$ and $D_{0}\left(\mathbf{q}, i q_{m}\right)=2 \omega_{\mathbf{q}}\left[\left(i q_{m}\right)^{2}-\omega_{\mathbf{q}}\right]^{-1}$ are the Matsubara Green's functions for noninteracting fermions and phonons, respectively, with $\omega_{n}$ and $q_{m}$ being fermion and boson Matsubara frequencies, which are odd and even multiples of $\pi / \beta=\pi k_{B} T$, respectively. Analytic continuation of $\Sigma\left(\mathbf{p}, i \omega_{n}\right)$ leads to the retarded self-energy $\Sigma^{R}(\mathbf{p}, \omega)=\Sigma\left(\mathbf{p}, i p_{n} \rightarrow \omega+i 0^{+}\right)$, which, at zero temperature and in the single (attractive) impurity limit, is given by

$$
\begin{aligned}
\Sigma^{R}(\mathbf{p}, \omega) & =U_{B F} n_{B}+\frac{1}{V} \sum_{\mathbf{q}} \times \\
& \left(\frac{g_{\mathbf{q}}^{2}}{\omega-\xi_{\mathbf{p}-\mathbf{q}}-\omega_{\mathbf{q}}+i 0^{+}}+\frac{m_{F B} U_{F B}^{2}}{2 n_{B}^{-1} m_{F} \epsilon_{\mathbf{q}}}\right) .
\end{aligned}
$$


In leading to Eq. (4), we have omitted an important step which we now explain. It goes back to Eqs. (2) and (3) where $U_{B F}$ should really be written as $U_{B F}^{\prime}$, a parameter introduced to model the Bose-Fermi interaction; our use of $U_{B F}$ instead of $U_{B F}^{\prime}$ is to simplify notation. $U_{B F}^{\prime}$ is connected to $U_{B F}$, up to the second order in $U_{B F}$, through [26]

$$
U_{B F}^{\prime}=U_{B F}+\frac{U_{B F}^{2}}{V} \sum_{\mathbf{q} \neq 0} \frac{m_{B F}}{q^{2}}
$$

according to the Lippmann-Schwinger equation for the two-body vacuum T-matrix [46] that describes the scattering between a boson and a fermion. Upon its substitution in Eq. (3), the linear term in Eq. (5) becomes the first term in Eq. (4) while the second order term in Eq. (5) is the origin of the last term in Eq. (4), which serves as a renormalization factor regulating the ultraviolet divergence known to exist in the integration for the self energy in Eq. (3).

This self-energy [Eq. (4)], which, strictly speaking, is valid only in the weak coupling regime, allows us to explore the subject in a conceptually intuitive and mathematically simplified manner. The ensuing results are expected to capture all qualitative features about the anisotropic polarons, provided that the impurity-phonon coupling is still much lower than the critical value for self-localization [25, 43, 44, 47, 48].

In what follows, we will use Eq. (4) or equivalently

$$
\begin{aligned}
\operatorname{Re} \Sigma^{R}(\mathbf{p}, \omega) & =U_{B F} n_{B}+\int \frac{d^{3} \mathbf{q}}{(2 \pi)^{3}} \times \\
& \left(\mathcal{P} \frac{g_{\mathbf{q}}^{2}}{\omega-\xi_{\mathbf{p}-\mathbf{q}}-\omega_{\mathbf{q}}}+\frac{m_{F B} U_{F B}^{2}}{2 n_{B}^{-1} m_{F} \epsilon_{\mathbf{q}}}\right),
\end{aligned}
$$

and

$$
\operatorname{Im} \Sigma^{R}(\mathbf{p}, \omega)=-\pi \int \frac{d^{3} \mathbf{q}}{(2 \pi)^{3}} g_{\mathbf{q}}^{2} \delta\left(\omega-\xi_{\mathbf{p}-\mathbf{q}}-\omega_{\mathbf{q}}\right),
$$

to study polarons in the dipolar condensate where $\mathcal{P}$ stands for the principal value.

\section{POLARON DESCRIPTION ON THE MASS SHELL AND ČERENKOV TYPE OF PHONON EMISSIONS}

In order to build some analytical insight, we first estimate the self-energy using the polaron energy $\omega$ on the mass shell, $\omega=\xi_{\mathbf{p}}$, and use this self-energy to characterize the polarons of interest. In this limit, we find that at small momenta where $\operatorname{Im} \Sigma^{R}\left(\mathbf{p}, \xi_{\mathbf{p}}\right)=0$, the impurity energy

$$
E_{\mathbf{p}}=\epsilon_{\mathbf{p}}+\operatorname{Re} \Sigma^{R}\left(\mathbf{p}, \xi_{\mathbf{p}}\right)
$$

is approximated as

$$
E_{\mathbf{p}} \approx E_{0}^{\star}+p_{\|}^{2} / 2 m_{F \|}^{\star}+p_{\perp}^{2} / 2 m_{F \perp}^{\star},
$$
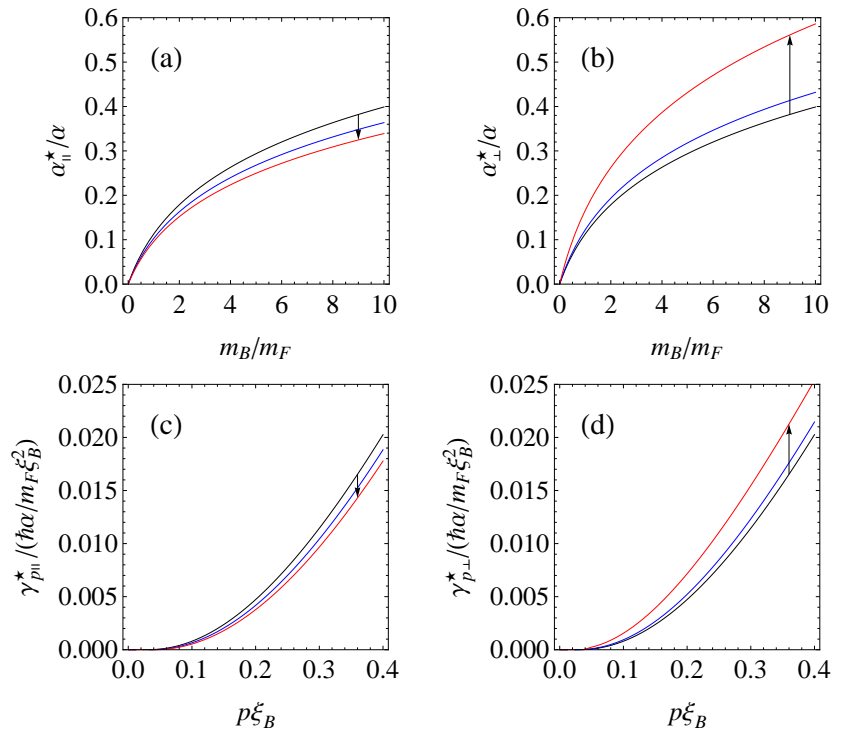

FIG. 2. (Color online) (a) $\alpha_{\|}$and (b) $\alpha_{\perp}$ as a function of $m_{B} / m_{F}$ for different $\varepsilon_{d d}=0$ (black), 0.3 (blue), and 0.8 (red). (c) $\gamma_{p_{\|}}^{\star}$ and (d) $\gamma_{p_{\perp}}^{\star}$ as a function of $p$ for different $\varepsilon_{d d}$ when $m_{B} / m_{F}$ is fixed to 21 . Note that because of the rich existence of atomic elements and their isotopes in nature, for practical purposes one can regard the mass ratio as a tunable parameter. The special case of $m_{B} / m_{F}=21$ in (c) and (d) corresponds to the mass ratio in the ${ }^{6} \mathrm{Li}$ and ${ }^{40} \mathrm{~K}^{86} \mathrm{Rb}$ mixture.

where $p_{\|}$and $p_{\perp}$ are, respectively, the momentum component along the dipole direction (axial momentum) and that normal to the dipole direction (radial momentum), and $m_{F||}^{\star}$ and $m_{F \perp}^{\star}$ are, respectively, the axial and radial effective polaron mass, the only two surviving elements in the mass tensor. Let $\bar{q}=q \xi_{B}, \bar{p}=p \xi_{B}, \bar{m}_{B}=m_{B} / m_{F}$, and $\bar{\omega}_{\mathbf{q}}=\omega_{\mathbf{q}} /\left(\hbar / m_{F} \xi_{B}^{2}\right)$ or

$$
\bar{\omega}_{\mathbf{q}}=\bar{q} \sqrt{1+\bar{q}^{2}+2 \varepsilon_{d d} P_{2}\left(z_{\mathbf{q}}\right)} /\left(2 \bar{m}_{B}\right)
$$

be the scaled quantities corresponding to $q, p, m_{B}$, and $\omega_{\mathbf{q}}$ in the so-called polaron unit system. Taylor expanding Eq. (6) up to the second order in the momenta, we find that

$$
m_{F||}^{\star}=m_{F} /\left(1-\alpha_{\|}^{\star}\right), \quad m_{F \perp}^{\star}=m_{F} /\left(1-\alpha_{\perp}^{\star}\right),
$$

where

$$
\begin{aligned}
& \alpha_{\|}^{\star}=2 \alpha_{\Sigma} \int d \bar{q} \int_{-1}^{+1} d z_{\mathbf{q}} \frac{\bar{q}^{6} z_{\mathbf{q}}^{2}}{\bar{\omega}_{\mathbf{q}}\left(\bar{\omega}_{\mathbf{q}}+\bar{q}^{2} / 2\right)^{3}}, \\
& \alpha_{\perp}^{\star}=\alpha_{\Sigma} \int d \bar{q} \int_{-1}^{+1} d z_{\mathbf{q}} \frac{\bar{q}^{6}\left(1-z_{\mathbf{q}}^{2}\right)}{\bar{\omega}_{\mathbf{q}}\left(\bar{\omega}_{\mathbf{q}}+\bar{q}^{2} / 2\right)^{3}} .
\end{aligned}
$$

In Eqs. (12),

$$
\alpha_{\Sigma}=\frac{\alpha}{32 \pi} \frac{1}{\bar{m}_{B}}\left(1+\frac{1}{\bar{m}_{B}}\right)^{2},
$$


is the coefficient expressed in terms of

$$
\alpha=a_{B F}^{2} /\left(a_{B B} \xi_{B}\right)
$$

which is a (dimensionless) polaronic coupling constant $[26,42]$. The ability to enhance $\alpha$ in Eq. (14) by tuning s-wave scattering lengths via Feshbach resonance [49-51] has been the main motivation behind the recent upsurge of activity in exploring polaronic physics in Bose-Fermi mixtures of cold atoms.

Figure 2 (a) and (b) illustrate how $\alpha_{\perp}$ and $\alpha_{\|}$change with the mass ratio, $m_{B} / m_{F}$, for different values of the dipolar interaction $\varepsilon_{d d}$. As a function of $m_{B} / m_{F}$ (for fixed $\left.\varepsilon_{d d}\right), \alpha_{\|}$behaves similarly to $\alpha_{\perp}$-both increase appreciably with $m_{B} / m_{F}$. As a function of $\varepsilon_{d d}$ (for fixed $\left.m_{B} / m_{F}\right), \alpha_{\|}$behaves oppositely to $\alpha_{\perp}$-as $\varepsilon_{d d}$ increases (but staying less than 1, beyond which the dipolar condensate is unstable), $\alpha_{\|}$decreases while $\alpha_{\perp}$ increases. To account for this anisotropy, we first note from Eq. (12) that contributions to $\alpha_{\|}$and $\alpha_{\perp}$ stem primarily from axial phonons (with $z_{\mathbf{q}}$ being close to 1 ) and radial phonons (with $z_{\mathbf{q}}$ being close to 0 ), respectively. In a dipolar condensate in which the attractive (head-to-tail) interaction competes with the repulsive (side-to-side) interaction, tuning the dipolar interaction $\varepsilon_{d d}$ (towards 1) serves to reduce the energy cost of the radial phonons while simultaneously increasing the energy cost of the axial phonons. As a result, at a very low impurity energy, such a tuning amounts to resonantly enhancing the interaction between the impurity and the radial phonon, on one hand, and off-resonantly suppressing the interaction between the impurity and the axial phonon, on the other hand. In turn, this results in $\alpha_{\|}$and $\alpha_{\perp}$ changing in the opposite directions in response to the change in the dipolar interaction, leading to the mass anisotropy in the polarons.

Let us now turn our attention to the imaginary part of the self-energy, $\operatorname{Im} \Sigma^{R}\left(\mathbf{p}, \xi_{\mathbf{p}}\right)$, on the mass shell and use it to obtain the spontaneous decay rate of an impurity polaron (in the sense of Fermi's golden rule), $\gamma_{\mathbf{p}}^{\star}=-$ $\operatorname{Im} \Sigma^{R}\left(\mathbf{p}, \xi_{\mathbf{p}}\right)$. Physically, when the imaginary part of the self-energy is non-vanishing, the energy of the impurity dissipates by spontaneous emission of phonons in the same sense of Cerenkov radiation [33, 34], where a charged particle emits electromagnetic radiation, when moving at a velocity higher than the phase velocity of light in a dispersive background medium. The speed of the phonon thus created depends on $z_{\mathbf{q}}$, the cosine of the angle between phonon momentum $\mathbf{q}$ and the dipole direction, according to

$$
v_{B}\left(z_{\mathbf{q}}\right)=\lim _{|\mathbf{q}| \rightarrow 0} \frac{\omega_{\mathbf{q}}}{|\mathbf{q}|}=v_{B} \sqrt{1+2 \varepsilon_{d d} P_{2}\left(z_{\mathbf{q}}\right)} .
$$

The momentum of the emitted phonon, dictated by energy conservation as represented by the Dirac $\delta$-function
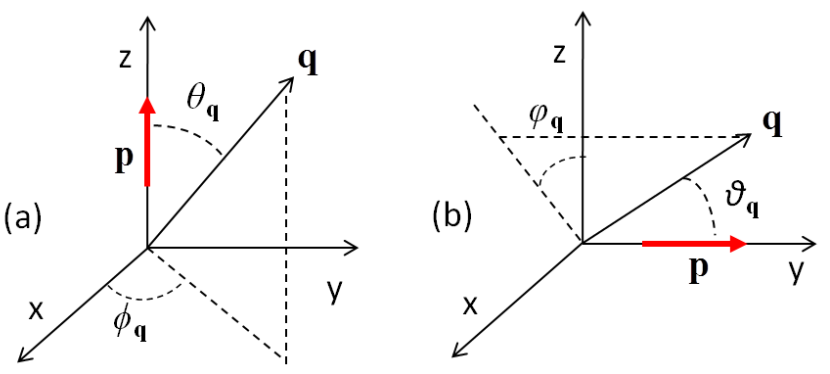

FIG. 3. (Color online) (a) The spherical coordinate system in which the zenith points to the $z$ axis and (b) the spherical coordinate system in which the zenith points to the $y$ axis. (a) and (b) are used to study phonon emissions by an impurity with momentum (thick red arrow) along the axial and radial directions, respectively.

in Eq. (7), has magnitude

$$
\begin{aligned}
\bar{q} & =\frac{2 \bar{p} y_{\mathbf{q}}}{1-\bar{m}_{B}^{-2}}- \\
& \frac{\sqrt{\left(2 \bar{p} y_{\mathbf{q}}\right)^{2}+\left(1-\bar{m}_{B}^{-2}\right)\left(1+2 \varepsilon_{d d} P_{2}\left(z_{\mathbf{q}}\right)\right)}}{\bar{m}_{B}\left(1-\bar{m}_{B}^{-2}\right)}
\end{aligned}
$$

which is a function of not only $z_{\mathbf{q}}$ but also $y_{\mathbf{q}}$, the cosine of the angle between phonon momentum $\mathbf{q}$ and impurity momentum p.

The anisotropy in the emitted phonons leads to the anisotropy in the decay rates. For an axial polaron, we use the usual spherical coordinate system in which the zenith points to the $z$ direction along which the axial impurity moves [Fig. 3(a)]. In this coordinate system, since both the momentum and dipoles are oriented along the $z$ direction, $z_{\mathbf{q}}=y_{\mathbf{q}}=\cos \theta_{\mathbf{q}}$, where $\left(\theta_{\mathbf{q}}, \phi_{\mathbf{q}}\right)$ are the usual polar and azimuthal angles. An analysis of $\operatorname{Im} \Sigma^{R}\left(\mathbf{p}, \xi_{\mathbf{p}}\right)$ then finds the axial decay rate to be given by

$$
\begin{array}{r}
\gamma_{p_{\|}}=\frac{\hbar}{m_{F} \xi_{B}^{2}} \pi \alpha_{\Sigma} \Theta\left(\bar{p}-\frac{\sqrt{1+2 \varepsilon_{d d}}}{2 \bar{m}_{B}}\right) \times \\
\int_{\cos \theta_{m}}^{1} \frac{\bar{q}^{5} d z_{\mathbf{q}}}{\left.\mid \bar{q}-\bar{p} y_{\mathbf{q}}\right) \bar{q} \omega_{\mathbf{q}}+\omega_{\mathbf{q}}^{2}+\frac{\bar{q}^{4}}{\left(2 \bar{m}_{B}\right)^{2}} \mid}
\end{array}
$$

where $\theta_{m}$ will be defined in Eq. (19). For a radial polaron, we adopt a different spherical coordinate system where the zenith points to the $y$ direction along which the radial impurity is assumed to move [Fig. 3(b)]. In such a coordinate system, $y_{\mathbf{q}}=\cos \vartheta_{\mathbf{q}}$ and $z_{\mathbf{q}}=\sin \vartheta_{\mathbf{q}} \cos \varphi_{\mathbf{q}}$, where $\left(\vartheta_{\mathbf{q}}, \varphi_{\mathbf{q}}\right)$ are the polar and azimuthal angle of this new coordinate system. A similar analysis of $\operatorname{Im} \Sigma^{R}\left(\mathbf{p}, \xi_{\mathbf{p}}\right)$ 
then finds the radial decay rate to be given by

$$
\begin{aligned}
\gamma_{p_{\perp}} & =\frac{\hbar}{m_{F} \xi_{B}^{2}} \pi \alpha_{\Sigma} \Theta\left(\bar{p}-\frac{\sqrt{1-\varepsilon_{d d}}}{2 \bar{m}_{B}}\right) \int_{0}^{2 \pi} \frac{d \varphi_{\mathbf{q}}}{2 \pi} \\
& \times \int_{\cos \vartheta_{m}}^{1} \frac{\bar{q}^{5} d y_{\mathbf{q}}}{\left(\bar{q}-\bar{p} y_{\mathbf{q}}\right) \bar{q} \bar{\omega}_{\mathbf{q}}+\bar{\omega}_{\mathbf{q}}^{2}+\frac{\bar{q}^{4}}{\left(2 \bar{m}_{B}\right)^{2}} \mid},
\end{aligned}
$$

where $\vartheta_{m}$ will be defined in Eq. (20). Again, $\bar{q}$ in both Eqs. (17) and (18) is defined in Eq. (16), and has the physical meaning of being the magnitude of the momentum of an emitted phonon. Figure 2 (c) and (d) plot $\gamma_{p_{\|}}^{\star}$ and $\gamma_{p_{\perp}}^{\star}$ as functions of $p$. The physical meanings of other terms are explained as follows.

The Heaviside step function, $\Theta(x)$, implements the Laudau criterion for superfluidity where the elementary excitations are the anisotropic phonons of a dipolar condensate. Thus, for an impurity traveling along the axial direction, dissipation takes place only when its velocity $v$ exceeds the sound velocity at $z_{\mathbf{q}}=1$ or $v_{B}(1)=v_{B} \sqrt{1+2 \varepsilon_{d d}}$ [Eq. (15)], a condition equivalent to $\bar{p}>\sqrt{1+2 \varepsilon_{d d}} / 2 \bar{m}_{B}$ in the polaron unit system. A similar analysis shows that for an impurity traveling along the radial direction where $z_{\mathbf{q}}=0$, dissipation takes place only when $\bar{p}>\sqrt{1-\varepsilon_{d d}} / 2 \bar{m}_{B}$.

The angles, $\theta_{m}$ in Eq. (19) and $\vartheta_{m}$ in Eq. (20), have the physical meaning that the phonon modes emitted by the supersonic impurity along the axial $(z)$ direction are within the cone of the polar angle (relative to the $z$-axis)

$$
\theta_{m}=\cos ^{-1} \sqrt{\frac{1-\varepsilon_{d d}}{\left(2 \bar{p} \bar{m}_{B}\right)^{2}-3 \varepsilon_{d d}}},
$$

and those emitted by the supersonic impurity along the radial direction are within the cone of the polar angle (relative to the $y$-axis)

$$
\vartheta_{m}=\cos ^{-1} \sqrt{\frac{1-\varepsilon_{d d}+3 \varepsilon_{d d} \sin ^{2} \varphi_{\mathbf{q}}}{\left(2 \bar{p} \bar{m}_{B}\right)^{2}+3 \varepsilon_{d d} \sin ^{2} \varphi_{\mathbf{q}}}} .
$$

As one may verify, the sine of the angle $\pi / 2-\theta_{m}$ $\left(\pi / 2-\vartheta_{m}\right)$ equals the ratio of the speed of sound to that of an axial (radial) polaron. Thus, by definition, $\pi / 2-\theta_{m}\left(\pi / 2-\vartheta_{m}\right)$ is the Mach angle, or equivalently $\cos \theta_{m}\left(\cos \vartheta_{m}\right)$ is the inverse of the Mach number [52], which is a figure of merit well-known in the study of shock waves, examples of which include sonic booms generated by supersonic flights [52], bow and stern waves created by high speed boats [53], Cerenkov radiation [33, 34] emitted by charged particles, and, more recently, Bogoliubov phonons emitted by atoms in superfluids [54-59]. In contrast to the axial impurity which preserves the cylindrical symmetry so that the cone of the polar angle $\theta_{m}$ [Eq. (19)] is independent of the azimuthal angle, the radial impurity breaks the cylindrical symmetry so that the cone of the polar angle $\vartheta_{m}$ [Eq. (20)] is now a function of azimuthal angle $\varphi_{\mathbf{q}}$, which can only occur in systems with anisotropy.
The invariance under Galilean transformations implies that one can test this anisotropy by letting a dipolar condensate move against a stationary impurity (for example, in the form of a localized optical potential of a far-detuned laser beam [60,61]). The emitted phonons are expected to form, behind the impurity, a conical wave front of fixed aperture $\pi / 2-\theta_{m}$ when moving along the axial direction and that of varying aperture $\pi / 2-\vartheta_{m}$ [in the fashion of Eq. (20)] when moving along the radial direction [35].

\section{SPECTRAL FUNCTIONS AND POLARONS ACCORDING TO FERMI LIQUID THEORY}

We propose to probe the polarons in BECs using the same rf spectroscopy that was successfully applied to explore the BEC-BCS crossover [62] in resonant Fermi gases [63] and fermionic polaron physics in highly imbalanced Fermi gases $[5,37]$. In rf spectroscopy, an rf field of amplitude $\Omega_{L}$ and frequency $\omega_{L}$ is applied to promote impurities to the excited (final) hyperfine state $|e\rangle$ that lies above the impurity state $|g\rangle$ by an energy $\hbar \omega_{e g}$, a process described by the Hamiltonian

$$
\hat{H}_{r f}=\frac{\hbar \Omega_{L}}{2} \int d^{3} \mathbf{r}\left[e^{-i \omega_{L} t} \hat{\psi}_{e}^{\dagger}(\mathbf{r}) \hat{\psi}_{g}(\mathbf{r})+h . c .\right],
$$

where $\hat{\psi}_{g, e}(\mathbf{r})$ are the relevant annihilation operators. The rf signal, which is the rate at which the population in the final state changes, is given by

$$
I=-2\left(\frac{\Omega_{L}}{2}\right)^{2} \operatorname{Im} \chi\left(\mu-\mu_{e}-\omega_{L}\right),
$$

within linear response theory (in which $\hat{H}_{r f}$ is treated as a small perturbation), where $\mu_{e}$ is the chemical potential of the final state and $\operatorname{Im} \chi(\omega)$ is the Fourier transform of the retarded time-ordered correlation function, $-i \Theta\left(t-t^{\prime}\right)\left\langle\left[\hat{\psi}_{e}^{\dagger}(\mathbf{r}, t) \psi_{g}(\mathbf{r}, t), \hat{\psi}_{g}^{\dagger}\left(\mathbf{r}^{\prime}, t^{\prime}\right) \psi_{e}\left(\mathbf{r}^{\prime}, t^{\prime}\right)\right]\right\rangle$, averaged over both $\mathbf{r}$ and $\mathbf{r}^{\prime}[64,65]$. In the case where the final state interaction is negligible, one can express, within the Matsubara (imaginary-time) formalism, the rf signal as $I(\omega) \propto \int d^{3} \mathbf{p} I_{0}(\mathbf{p}, \omega)$ in terms of the momentumresolved current

$$
I_{0}(\mathbf{p}, \omega)=\left(\frac{\Omega_{L}}{2}\right)^{2} A\left(\mathbf{p}, \xi_{\mathbf{p}}-\omega\right) f\left(\xi_{\mathbf{p}}-\omega\right),
$$

where $\omega=\omega_{L}-\omega_{e g}$ is the rf detuning, $f(x)=\left(e^{\beta x}+1\right)^{-1}$ is the Fermi distribution function which becomes a step function $\Theta(-x)$ in the limit of zero temperature, and $A(\mathbf{p}, \omega)$ is the spectral function satisfying the sum rule $\int_{-\infty}^{+\infty} d \omega A(\mathbf{p}, \omega) / 2 \pi=1$. The momentum resolved rf spectroscopy $[36,37]$ is the cold-atom analog of the angle resolved photoemission spectroscopy in solid state physics [66]. It measures $I_{0}(\mathbf{p}, \omega)$ and thus allows the spectral function, $A(\mathbf{p}, \omega)=-2 \operatorname{Im} G\left(\mathbf{p}, \omega+i 0^{+}\right)$, to 

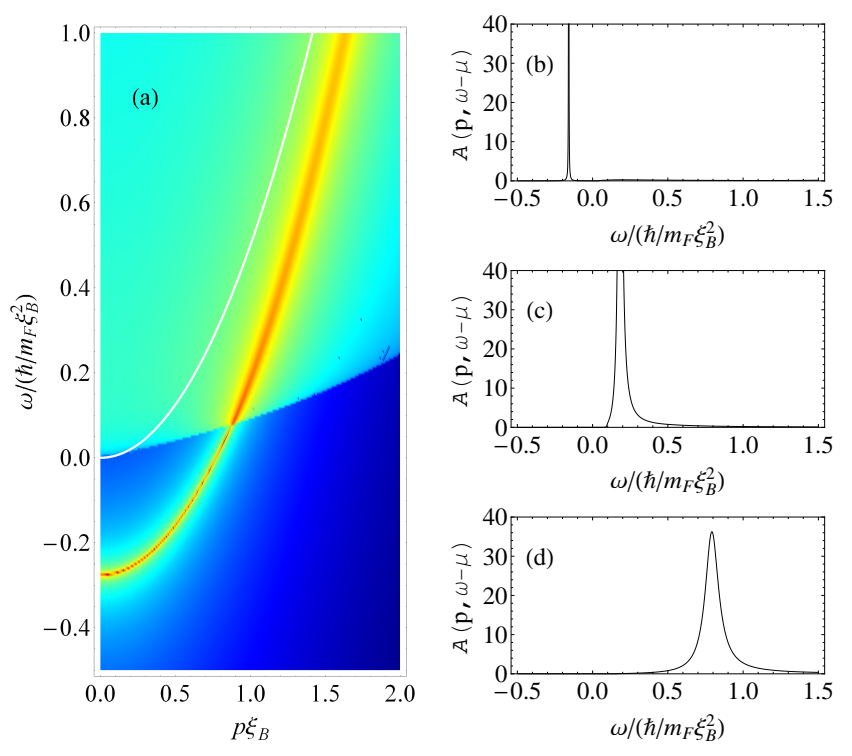

FIG. 4. (Color online) (a) The contour plot of the spectral function $[\log (A(\mathbf{p}, \omega-\mu))]$ of axial plarons in the $(p, \omega)$ space. The free Fermi dispersion relation is plotted as a thin white line. The figures on the right panel show how the spectral function change with $\omega$ for polarons with axial momentum of $0.5(\mathrm{~b}), 1$ (c), and $1.5(\mathrm{~d})$. The parameters used are $\varepsilon_{d d}=0.95$, $m_{F}=6 u, m_{B}=10 m_{F}, a_{B B}=200 a_{0}, a_{B F}=-500 a_{0}$, $n_{B}=10^{20} \mathrm{~m}^{-3}$, where $u$ is the atomic mass and $a_{0}$ is the Bohr radius. Note that in this work, we are interested in the physics of mobile impurities. Thus, $\alpha$ is chosen to be below the critical value for self localization. In the considered example, $\alpha=0.48$. In producing these diagrams, a small but finite number is used to approximate $0^{+}$.

be accessed experimentally, where $G\left(\mathbf{p}, i \omega_{n}\right)$ is the Matsubara Green's function determined by Dyson's equation $G^{-1}\left(\mathbf{p}, i \omega_{n}\right)=G_{0}^{-1}\left(\mathbf{p}, i \omega_{n}\right)-\Sigma\left(\mathbf{p}, i \omega_{n}\right)$. In terms of the retarded self-energy, the spectral function $A(\mathbf{p}, \omega)$ takes the form

$$
A(\mathbf{p}, \omega)=\frac{-2 \operatorname{Im} \Sigma^{R}(\mathbf{p}, \omega)}{\left[\omega-\xi_{\mathbf{p}}-\operatorname{Re} \Sigma^{R}(\mathbf{p}, \omega)\right]^{2}+\operatorname{Im} \Sigma^{R}(\mathbf{p}, \omega)^{2}} .
$$

Figure 4(a) showcases a typical contour plot of $A(\mathbf{p}, \omega-\mu)$ for an axial impurity. The key features of this diagram can be captured in terms of the Fermi-liquid parameters. The peak position, $E_{\mathbf{p}}$, is the root to the equation,

$$
E_{\mathbf{p}}-\mu=\xi_{\mathbf{p}}+\operatorname{Re} \Sigma^{R}\left(\mathbf{p}, E_{\mathbf{p}}-\mu\right)
$$

involving the real part of the self-energy. In the region close to $\mathbf{p}=0$, the peak position is approximated as a quadratic function of momenta, $E_{\mathbf{p}} \approx E_{0}+$ $p_{\|}^{2} / 2 m_{F \|}^{*}+p_{\perp}^{2} / 2 m_{F \perp}^{*}$. The curvature close to $\mathbf{p}=0$ is inversely proportional to the effective mass, $m_{F \|, \perp}^{*}=$ $m_{F} Z_{0}^{-1}\left(1+\partial_{\epsilon_{p_{\|}, \perp}} \operatorname{Re} \Sigma^{R}(\mathbf{p}, \omega)\right)^{-1}$, where $Z_{0}=(1-$ $\left.\partial_{\omega} \operatorname{Re} \Sigma^{R}(\mathbf{p}, \omega)\right)^{-1}$ is the quasiparticle residue, $\epsilon_{p_{\|, \perp}}=$ $p_{\|, \perp}^{2} / 2 m_{F}$ is the kinetic energy, and every term in $m_{F \|, \perp}^{*}$ is evaluated at $\mathbf{p}=\omega=0$.

The $\omega$-intercept of the peak line in Fig. 4(a) with the vertical axis determines the ground state polaron energy $E_{0}$. In the single polaron limit, $E_{0}=\mu$, where the chemical potential, $\mu$, is determined self consistently from $\mu=\operatorname{Re} \Sigma(\mathbf{0}, 0)[67]$ or

$$
\mu=U_{B F} n_{B}+\int \frac{d^{3} \mathbf{q}}{(2 \pi)^{3}}\left(\frac{g_{\mathbf{q}}^{2}}{\mu-\epsilon_{\mathbf{q}}-\omega_{\mathbf{q}}}+\frac{m_{F B} U_{F B}^{2}}{2 n_{B}^{-1} m_{F} \epsilon_{\mathbf{q}}}\right) .
$$

It has been well established that highly imbalanced Fermi systems support not only attractive [3-6] but also repulsive [68-72] polarons. Likewise, repulsive polarons are also expected to exist in our system when $U_{B F}$ is tuned on the repulsive side of the Feshbach resonance, but the repulsive polarons are highly unstable [73]. In this work, consistent with the assumption that led to the self-energy in Eq. (4), we limit our investigation to systems with attractive Bose-Fermi interaction $\left(U_{B F}<0\right)$ where polarons are always attractive. The $\omega$-intercept in Fig. 4(a) indicates a polaron energy (or rather a binding energy) of $E_{0}=-0.276\left(\hbar^{2} / m_{F} \xi_{B}^{2}\right)$, which is in agreement with the one determined from Eq. (26).

The existence of a negative polaron energy creates, for small momenta, a gap between the polaron peak dispersion curve and the impurity-phonon scattering continuum (light blue region), a region defined to be above $\omega_{c}(\mathbf{p})=\min \left(\epsilon_{\mathbf{p}-\mathbf{q}}+\omega_{\mathbf{q}}\right)$, where $\mathbf{q}$ covers all the possible phonon momenta. For an impurity with a momentum below a certain threshold $p_{c}[\approx 0.88$ in Fig. 4(a)], the impurity-phonon interaction is coherent, and the spectral function describing the peak is then a $\delta$-like function as in the case of free fermions. For an impurity with a momentum above $p_{c}$ the polaron peak line enters the continuum where the phonon states become energetically available for impurity scattering (and at very large momenta, as expected, the polaron peak line asymptotes to the free impurity dispersion). The impurity scattering involving the phonon emissions then causes the peak line to be broadened as illustrated in diagrams on the right panel of Fig. 4.

It is well-known that the gap shown in Fig. 4(a) can cause the calculation using Fermi liquid theory (equivalent to the Tamm-Dancoff approximation) to be quite different from the calculation using the on-mass-shell approximation (also known as the Rayleigh-Schrödinger approximation) [74]. In the present problem, this same gap has an added consequence of diminishing the role that anisotropy plays in Fermi liquid theory. In a future study, we plan to tailor Feynman's path integral formalism [75] for exploring the polaron physics in our model; as a superior all coupling approximation, Feynman's method would not only extend the current study to the strong-coupling regime, but would also help arbitrate between the two approaches just mentioned. 


\section{CONCLUSION}

In conclusion, we have considered a polaronic model in which impurity fermions interact, via the short-range s-wave scattering potential, with background bosons in a dipolar condensate where bosons interact via both shortand long-range interactions. We have described such a model with a Fröhlich type of Hamiltonian where the role of phonons is played by density fluctuations of dipolar bosons. The polaron in this model emerges as an impurity dressed with a cloud of phonons which, due to the competition between the attractive and repulsive part of the dipole-dipole interaction, obey an anisotropic dispersion spectrum. Taking into consideration only the single-phonon-impurity scattering processes, we have constructed a self-energy capable of capturing the main polaronic features of our model operating in the weak coupling regime. We have described the polaron using the self-energy on the on-mass-shell, discussing, in particular, how anisotropy affects the Čerenkov radiation of Bogoliubov phonon modes, which can be directly verified by experiments in which a dipolar BEC moves against an obstacle. We have also described the polaron in the spirit of Fermi liquid theory, focusing on the spectral function for the impurity fermions, which is directly accessible to the momentum resolved rf spectroscopy in cold atoms. Finally, we stress that while this work concentrates on the three-dimensional dipolar Bose-Fermi mixture, it can be generalized to its two-dimensional analog where the polaron physics may be greatly enriched by the existence of a "roton" minimum structure in the phonon spectrum of the dipolar BEC $[76,77]$.

Note added: While this work was under review, we were informed of the article [78] by its authors, which uses a variational approach to calculate the spectral properties and rf spectroscopy of polarons in a nondipolar BEC.

\section{ACKNOWLEDGEMENTS}

H. Y. L. is supported in part by the US Army Research Office under Grant No. W911NF-10-1-0096 and in part by the US National Science Foundation under Grant No. PHY11-25915.
[1] F. Chevy and C. Mora, Rep. Prog. Phys. 73, 112401 (2010).

[2] P. Massignan, M. Zaccanti, and G. M. Bruum, arXiv:1309.0219.

[3] N. Prokof'ev and B. Svistunov, Phys. Rev. B 77, 020408 (2008).

[4] C. Mora and F. Chevy, Phys. Rev. A 80, 033607 (2009).

[5] A. Schirotzek, C.-H. Wu, A. Sommer, and M. W. Zwierlein, Phys. Rev. Lett. 102, 230402 (2009).

[6] S. Nascimbène, N. Navon, K. J. Jiang, L. Tarruell, M. Teichmann, J. McKeever, F. Chevy, and C. Salomon, Phys. Rev. Lett. 103, 170402 (2009).

[7] P. W. Anderson, "A Career in Theoretical Physics," 2nd ed., World Scientific Publishing Company, Singapore (2005).

[8] A. C. Hewson, "The Kondo Problem to Heavy Fermions," Cambridge University Press, Cambridge (1997).

[9] L. D. Laudau, Phys. Z. Sowjetunion 3, 644 (1933).

[10] N. Mannella, W. L. Yang, X. J. Zhou, H. Zheng, J. F. Mitchell, J. Zaanen, T. P. Devereaux, N. Nagaosa, Z. Hussain, and Z. X. Shen, Nature 438, 474 (2005).

[11] A. Lanzara, P. V. Bogdanov, X. J. Zhou, S. A. Kellar, D. L. Feng, E. D. Lu, T. Yoshida, H. Eisaki, A. Fujimori, K. Kishio, et al., Nature 412, 510 (2001).

[12] P. A. Lee, N. Nagaosa, and X.-G. Wen, Rev. Mod. Phys. 78, 17 (2006).

[13] J. T. Devreese and A. S. Alexandrov, Rep. Prog. Phys. 72, 066501 (2009).

[14] A. S. Alexandrov, Phys. Rev. B 77, 094502 (2008).

[15] K.-K. Ni, S. Ospelkaus, M. H. G. de Miranda, A. Pe'er, B. Neyenhuis, J. J. Zirbel, S. Kotochigova, P. S. Julienne, D. S. Jin, and J. Ye, Science 322, 231 (2008).
[16] S. Ospelkaus, K.-K. Ni, M. H. G. de Miranda, B. Neyenhuis, D. Wang, S. Kotochigova, P. S. Julienne, D. S. Jin, and J. Ye, Faraday Discuss. 142, 351 (2009).

[17] M. Vengalattore, S. R. Leslie, J. Guzman, and D. M. Stamper-Kurn, Phys. Rev. Lett. 100, 170403 (2008).

[18] J. Stuhler, A. Griesmaier, T. Koch, M. Fattori, T. Pfau, S. Giovanazzi, P. Pedri, and L. Santos, Phys. Rev. Lett. 95, 150406 (2005).

[19] M. Lu, N. Q. Burdick, S. H. Youn, and B. L. Lev, Phys. Rev. Lett. 107, 190401 (2011).

[20] K. Aikawa, A. Frisch, M. Mark, S. Baier, A. Rietzler, R. Grimm, and F. Ferlaino, Phys. Rev. Lett. 108, 210401 (2012).

[21] L. Santos, G. V. Shlyapnikov, P. Zoller, and M. Lewenstein, Phys. Rev. Lett. 85, 1791 (2000).

[22] S. Yi and L. You, Phys. Rev. A 61, 041604 (2000).

[23] L. Lahaye, C. Menotti, L. Santos, M. Lewenstein, and T. Pfau, Rep. Prog. Phys. 72, 126401 (2009).

[24] G. Bismut, B. Laburthe-Tolra, E. Maréchal, P. Pedri, O. Gorceix, and L. Vernac, Phys. Rev. Lett. 109, 155302 (2012).

[25] F. M. Cucchietti and E. Timmermans, Phys. Rev. Lett. 96, 210401 (2006).

[26] J. Tempere, W. Casteels, M. K. Oberthaler, S. Knoop, E. Timmermans, and J. T. Devreese, Phys. Rev. B 80, 184504 (2009).

[27] W. Casteels, J. Tempere, and J. T. Devreese, Phys. Rev. A 83, 033631 (2011).

[28] M. Bruderer, A. Klein, S. R. Clark, and D. Jaksch, Phys. Rev. A 76, 011605 (2007).

[29] A. Privitera and W. Hofstetter, Phys. Rev. A 82, 063614 (2010).

[30] A. P. Chikkatur, A. Görlitz, D. M. Stamper-Kurn, S. Inouye, S. Gupta, and W. Ketterle, Phys. Rev. Lett. 85, 
$483(2000)$.

[31] J. Catani, G. Lamporesi, D. Naik, M. Gring, M. Inguscio, F. Minardi, A. Kantian, and T. Giamarchi, Phys. Rev. A 85, 023623 (2012).

[32] R. Scelle, T. Rentrop, A. Trautmann, T. Schuster, and M. K. Oberthaler, Phys. Rev. Lett. 111, 070401 (2013).

[33] P. A. Cerenkov, Doklady Akaddemii Nauk SSSR 2, 451 (1934).

[34] P. A. Cerenkov, Phys. Rev. 52, 378 (1937).

[35] P. Muruganandam and S. Adhikari, Physics Letters A 376, 480 (2012), ISSN 0375-9601.

[36] J. T. Stewart, J. P. Gaebler, and D. S. Jin, Nature 454, 744 (2008).

[37] M. Feld, B. Froöhlich, E. Vogt, M. Koschorreck, and M. Köhl, Nature 480, 75 (2011).

[38] K. Góral, K. Rzaz̧żewski, and T. Pfau, Phys. Rev. A 61, 051601 (2000).

[39] S. Giovanazzi, A. Görlitz, and T. Pfau, Phys. Rev. Lett. 89, 130401 (2002).

[40] C. Kittel, "Introduction to Solid State Physics," John Wiley and Sons, Inc., New York (1986).

[41] F. M. Peeters and J. T. Devreese, Phys. Rev. B 32, 3515 (1985).

[42] W. Casteels, J. Tempere, and J. T. Devreese, Phys. Rev. A 84, 063612 (2011).

[43] D. H. Santamore and E. Timmermans, New J. of Phys. 13, 103029 (2011).

[44] A. A. Blinova, M. G. Boshier, and E. Timmermans, arXiv:1304.7704 (2013).

[45] W. Casteels, J. Tempere, and J. T. Devreese, Phys. Rev. A 86, 043614 (2012).

[46] A. L. Fetter and J. D. Walecka, "Quantum Theory of Many-Particle Systems," McGraw-Hill Book Company, New York (1994).

[47] R. M. Kalas and D. Blume, Phys. Rev. A 73, 043608 (2006).

[48] M. Bruderer, W. Bao, and D. Jaksch, Eur. Phys. Lett. 82, 30004 (2008).

[49] E. Tiesinga, B. J. Verhaar, and H. T. C. Stoof, Phys. Rev. A 47, 4114 (1993).

[50] S. Inouye, M. R. Andrews, J. Stenger, H.-J. Miesner, D. M. Stamper-Kurn, and W. Ketterle, Nature 392, 151 (1998).

[51] E. Timmermans, P. Tommasini, M. Hussein, and A. Kerman, Phys. Rep. 315, 199 (1999)

[52] E. Mach and P. Salcher, Sitzungsberichte (Viena) 95, 764 (1887).

[53] G. B. Whitman, "Linear and Nonlinear Waves," Wiley, New York (1974).
[54] B. Damski, Phys. Rev. A 69, 043610 (2004).

[55] I. Kulikov and M. Zak, Phys. Rev. A 67, 063605 (2003).

[56] A. M. Kamchatnov, A. Gammal, and R. A. Kraenkel, Phys. Rev. A 69, 063605 (2004).

[57] L. Salasnich, N. Manini, F. Bonelli, M. Korbman, and A. Parola, Phys. Rev. A 75, 043616 (2007).

[58] I. Carusotto, S. X. Hu, L. A. Collins, and A. Smerzi, Phys. Rev. Lett. 97, 260403 (2006).

[59] Y. G. Gladush, G. A. El, A. Gammal, and A. M. Kamchatnov, Phys. Rev. A 75, 033619 (2007).

[60] A. Amo, S. Pigeon, D. Sanvitto, V. G. Sala, R. Hivet, I. Carusotto, F. Pisanello, G. Leménager, R. Houdré, E. Giacobino, et al., Science 332, 1167 (2011).

[61] Talk given by E. Cornell at the KITP Conference on Quantum Gases (University of California, Santa Barbara, 2004). Available at http://online.itp.ucsb.edu/online/gases_c04/cornell.

[62] C. Chin, M. Bartenstein, A. Altmeyer, S. Riedl, S. Jochim, J. H. Denschlag, and R. Grimm, Science 305, 1128 (2004).

[63] J. Kinnunen, M. Rodriguez, and P. Törmä, Science 305, 1131 (2004).

[64] M. Punk and W. Zwerger, Phys. Rev. Lett. 99, 170404 (2007).

[65] P. Massignan, G. M. Bruun, and H. T. C. Stoof, Phys. Rev. A 77, 031601 (2008).

[66] A. Damascelli, Z. Hussain, and Z.-X. Shen, Rev. Mod. Phys. 75, 473 (2003).

[67] R. Combescot, A. Recati, C. Lobo, and F. Chevy, Phys. Rev. Lett. 98, 180402 (2007).

[68] X. Cui and H. Zhai, Phys. Rev. A 81, 041602 (2010).

[69] P. Massignan and G. M. Bruun, Eur. Phys. J. D. 65, 83 (2011).

[70] R. Schmidt and T. Enss, Phys. Rev. A 83, 063620 (2011).

[71] M. Koschorreck, D. Pertot, E. Vogt, B. Fröhlich, M. Feld, and M. Köhl, Nature 485, 619 (2012).

[72] C. Kohstall, M. Zaccanti, M. Jag, A. Trenkwalder, P. Massignan, G. M. Bruun, F. Schreck, and R. Grimm, Nature 485, 615 (2012).

[73] S. P. Rath and R. Schmidt, arXiv:1308.3457 (2013).

[74] G. D. Mahan, "Many-Particle Physics," 3rd ed., Kluwer Academic/Plenum Publishers, New York (2000).

[75] R. P. Feynman, Phys. Rev. 97, 660 (1955).

[76] L. Santos, G. V. Shlyapnikov, and M. Lewenstein, Phys. Rev. Lett. 90, 250403 (2003).

[77] C. Ticknor, R. M. Wilson, and J. L. Bohn, Phys. Rev. Lett. 106, 065301 (2011).

[78] A. Shashi, F. Grusdt, D. A. Abanin, and E. Demler, arXiv:1401.0952. 